\title{
Research of Partition Function on Optical Properties and Temperature Diagnosis of Air Plasma
}

\author{
Dechuan Qiu, Guoqiang Gao*, Wenfu Wei*, Haixing Hu, Chunmao LI, Guangning Wu \\ School of Electrical Engineering, Southwest Jiaotong University, Chengdu, Sichuan 610031, China \\ *Corresponding author: xnjdggq@ 163.com; wfwei@home.swjtu.edu.cn
}

Manuscript Number MB-1481R1

\begin{abstract}
The relationship between partition function, particle density, refractive index, and temperature for atmospheric plasma is calculated based on thermodynamics and chemical equilibrium. Taking into account the contribution of hydrogen-like levels to the atomic partition function, a compact method to calculate the atomic partition function is first used with the Eindhoven model to deduce the plasma's refractive index. Results calculated by the new approach and two other traditional simplified methods are compared and analyzed. For a better understanding on the temperature measurement accuracy deduced by different partition function disposal approaches, moiré deflectometry is employed as the experimental scheme to acquire the refractive index-position curve. Finally, applicability of different partition function disposal approaches are discussed, and results indicate that the optical properties deduced in this paper are well suited for the refractive index-based plasma diagnosis.
\end{abstract}

Keywords:

Air plasmas; partition functions; plasma component; refractive index; moiré deflectometry; temperature distribution

\section{Introduction}

Since plasma was first defined as the fourth state of matter by William Crooks in 1879, a large amount of applications have been found in the science, technology, and industry areas [1,2]. Particularly, atmospheric plasmas are receiving more and more attention due to the advantages of low cost, simple set-up, lack of need to construct a vacuum chamber, etc. [3]. Current interest in atmospheric plasmas are related to ozone production, water treatment, material processing, and more [4]. For further development of atmospheric plasmas in different fields, a better understanding of the plasmas fundamentals is always desirable. Temperature has been widely accepted as one of the key parameters that indicates plasma properties, while optical properties play an important role in the results interpretation of the refractive index-based plasma diagnosis.

To study the plasma's temperature and optical properties, methods like emission spectrum, laser interferometry, and moiré deflectometry have been adopted extensively.
Compared with other experimental methods, moiré deflectometry has its own advantages, such as low cost and low interference in the flow field [5,6]. However, optical diagnostic techniques always rely on deducing and interpreting the refractive index to acquire the plasma's temperature. Refractive index is generally regarded as a function of the number density related to the multiple components of neutral particle, ion, and electron respectively. Thus, solving the component of plasma appropriately is always a preliminary step for researchers.

Currently Chen yun-yun and Fatemeh have already done some research on the relationship between plasma's temperature and optical properties [3,5,6,7]. Both of them adopted the Eindhoven model as the computing approach to solve plasma's component instead of the models based on chemical kinetics or minimum Gibbs free enthalpy adopted in references $[8,9]$. The reason for choosing the Eindhoven model is primarily attributed to its accuracy, which is comparable to the other two methods when plasma is in local 
temperature equilibrium (LTE) status, while the parameters used in the Eindhoven model are much easier to acquire. However, a very important parameter called partition function, which affects the calculated component results, has been adopted in simplified form in previous works. Some literature neglected the hydrogen-like levels of the atomic particles, while others treated the partition function's ratio as a constant unit. These two partition function disposal approaches significantly reduce the complexity of the Eindhoven model and conspicuously save the running time of the program. But meanwhile they inevitably bring in the inaccurate factors to the plasma's concentration solved by the Eindhoven model, which would further influence the refractive index result. Attention should be paid to the above-mentioned issues.

Recently a series of works published by Giuliano have provided a new compact model to calculate the partition function [10,11,12]. This new partition function disposal method avoids consuming lots of time on the cut-off criterion and successfully retains accuracy at the same time. It gives us a new way to solve the Eindhoven model. Correspondingly, an extension of this method to further obtain information on plasmas, including components and refractive index, would be highly desirable.

In this paper three different partition function disposal methods are adopted to calculate the air plasma's component separately. The plasma's concentration is used to compute the refractive index. Meanwhile, moiré deflectometry is employed as the experimental scheme to deduce the refractive index of typical discharge-produced plasma. The temperature distribution is acquired from the inversion of the refractive index, and the differences among the outcome deduced from the three partition function disposal methods are compared and discussed.

\section{Theoretical Analysis and Results}

The flowchart for the calculation of optical properties and the diagnosis of temperature distribution is given in Fig. 1. The Eindhoven model is employed to obtain the plasma's composition, which provides the basis for the calculation of the distribution of the refractive index versus temperature. Moiré deflectometry is employed to obtain the spatial refractive index distribution. As a result, the spatial temperature distribution could be achieved through figuring out the relationship of the refractive index versus temperature. To investigate the influences of the partition functions on the calculated results of composition and optical properties, three different partition function disposal methods are employed separately in the Eindhoven model. The composition is computed by the Newton iterative method, and the distribution of the refractive index versus temperature is deduced from the particle number density of electron, ion, and neutral particles.

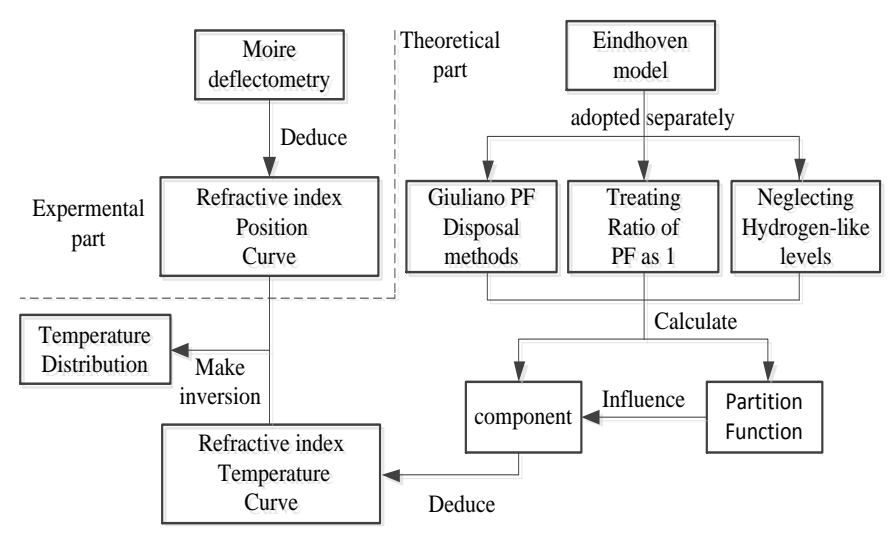

Fig. 1. The flowchart for researching the influence of partition function to the optical property and diagnosis of temperature distribution

\subsection{Eindhoven Model}

The Eindhoven model is used for calculating plasma's component, which is based on thermodynamics and statistical physics $[13,14,15]$. It is made up of four laws, respectively named mass action law, plasma quasi-neutral condition, Dalton's law, and stoichiometric balance of concentration [9]. In the calculation of the composition, the species including $\mathrm{N}_{2}, \mathrm{~N}_{2}^{+}, \mathrm{N}, \mathrm{N}^{1+}, \mathrm{N}^{2+}, \mathrm{N}^{3+}, \mathrm{O}_{2}, \mathrm{O}_{2}^{+}, \mathrm{O}, \mathrm{O}^{1+}, \mathrm{O}^{2+}, \mathrm{O}^{3+}$, and free electrons are taken into account. The specific forms of mass action law are Guldberg-Waage equations describing the dissociation process, and Saha equations describing the ionization process. Guldberg-Waage equations can be manifested as follows:

$$
\frac{n_{A} n_{B}}{n_{C}}=\frac{Z_{\text {int }, A} Z_{\text {int }, B}}{Z_{C}}\left(\frac{2 \pi m_{A} m_{B} k T_{h}}{m_{C} h^{2}}\right)^{\frac{3}{2}} e^{\left(-\frac{E_{d}}{k T_{h}}\right)}
$$

where $n_{r}, m_{r}$, and $Z_{\text {int, } r}$ are concentration, mass, and internal partition function of $r$ th particle respectively; $h$ and $\kappa$ are the 
Plank and Boltzmann constants. $E_{d}$ is the dissociation energy, and $T_{h}$ is the heavy particle temperature of plasma. Saha equations can be written as:

$$
\frac{n_{r+1} n_{e}}{n_{r}}=\frac{2 Z_{\text {int }, r+1}}{Z_{\text {int }, r}}\left(\frac{2 \pi m_{e} k T_{e}}{h^{2}}\right)^{\frac{3}{2}} e^{\left(-\frac{E_{r}-\Delta E_{r}}{k T_{e x}}\right)}
$$

where $T_{e}, T_{e x}, E_{r}$, and $\Delta E_{r}$ are the electron temperature, the excitation temperature, the ionization energy, and the ionization potential lowing (I.P.L) of $r$ th particle respectively. In two-temperature plasma, $T_{e x}$ could be considered approximately equal to $T_{e}$. When plasma is in LTE status, $T_{h}$ is equal to $T_{e}$ as well as $T_{e x}$. The I.P.L is always equal to zero in dissociation reactions. There are a few theories for $\Delta E_{r}$ 's calculation. In this paper we adopt the well accepted scheme from Griem [16], which is written as follows:

$$
\Delta E_{r}=\frac{\left(Z_{r}+1\right) e_{C}^{2}}{4 \pi \varepsilon_{0} \lambda_{D}}
$$

where $e_{c}, \varepsilon_{0}$, and $\lambda_{D}$ are the electron charge, the permittivity of vacuum, and the Debye distance respectively.

The formula of the plasma quasi-neutral condition is:

$$
\sum_{l} q_{l} n_{l}^{+}=\sum_{j} q_{j} n_{j}^{-}+n_{e}
$$

where $l$ and $j$ are the species of positive ion and negative ion separately. Dalton's law manifests that the mixed gas's total pressure is equal to the summation of each component's own pressure. Pressure correction is taken into consideration in the analysis to guarantee the accuracy of when electron density is high. The law then can be written as:

$$
P=\left(n_{e}+\sum_{r=1}^{s} n_{r}\right) k T-\Delta P
$$

where $s$ is the total species of the ions, and $\Delta P$ denotes the pressure correction due to Coulombian interactions. It can be written as [15]:

$$
\Delta P=\frac{1}{24 \pi \varepsilon_{0} \lambda_{d}} \sum_{i=1}^{n} Z_{i}^{2} n_{i}
$$

Finally, the expression of the stoichiometric balance condition is given as:

$$
\frac{\sum_{s} n_{s}}{\sum_{p} n_{p}}=C
$$

where $s$ and $p$ are the total species of the relative element, and
$C$ is a constant.

After solving the Eindhoven model, the particle density could be used to calculate the refractive index. The refractive index's expression of plasma could be expressed as:

$$
n-1=\frac{1}{L}\left(A+\frac{B}{\lambda^{2}}\right)\left(N_{n}+\eta N_{i}\right)-4.46 * 10^{-14} \lambda^{2} N_{e}
$$

where $N_{n}, N_{i}$, and $N_{e}$ are the number density of neutral particle, ion, and electron respectively. $L$ and $\lambda$ are the Loschmidt number and probe wavelength respectively. $A$ and $B$ are constants relevant to molecules and atoms for specific components [3].

\subsection{Partition Function}

Because calculation of composition is related to partition function $(\mathrm{PF})$, while refractive index is dependent on the number density of different compositions according to Eq. (8), $\mathrm{PF}$ should be calculated in an appropriate form. Current atomic PF methods either adopt cut-off criterion, which has a fussy procedure in screening the satisfied levels, or oversimplify the hydrogen-like levels, which brings in inaccurate factors to PF's value. Thus, in the next part we adopt a new atomic PF disposal method, which avoids the two disadvantages mentioned above. For comparison with the existing literature and our outcome, we also calculate the PF's value by adopting two simplified methods, which either neglect the hydrogen-like levels or treat the PF's ratio as 1 .

\subsubsection{Atomic Internal Partition Function}

To solve the problems in cut-off criterion and simplified PF calculation, Giuliano's three lumped levels model is used to calculate the partition functions' value [10]. The expression of Giuliano's PF disposal method is given as:

$$
Z_{\text {int }}=G_{g}+G_{l} e^{\left(-\frac{E_{l}}{k T}\right)}+G_{h}(\Delta E) e^{\left(-\frac{E_{h}}{k T}\right)}
$$

where $G_{g}, G_{l}$, and $G_{h}(\Delta E)$ are the statistical weight of the ground, low levels, and hydrogen-like levels, with $E_{l}$ and $E_{h}$ referred to as the energy of low levels and hydrogen-like levels. Partial parameters of (9) are summarized in table I, where $g_{\text {core }}$ is a constituent part of $G_{h}$. The data we adopted came from the newest NIST atomic spectra database (version 5.3) [17]. 
Table I. The Parameters of Three Lumped Levels Belong to the N(I)-N(IV) Ions.

\begin{tabular}{|c|c|c|c|c|}
\hline Ion & $\mathrm{G}_{0}$ & $\mathrm{G}_{1}$ & $\begin{array}{l}\text { First excited } \\
\text { configuration }\end{array}$ & $\mathrm{g}_{\text {core }}$ \\
\hline $\mathrm{N}(\mathrm{I})$ & 4 & 16 & $\begin{array}{l}1 s^{2} 2 S^{2} 2 P^{3}\left({ }^{2} D^{\circ}\right) \\
1 s^{2} 2 S^{2} 2 P^{3}\left({ }^{2} P^{\circ}\right)\end{array}$ & 9 \\
\hline \multirow[b]{2}{*}{$\mathrm{N}(\mathrm{II})$} & \multirow[b]{2}{*}{9} & 11 & $\begin{array}{l}1 \mathrm{~s}^{2} 2 \mathrm{~S}^{2} 2 \mathrm{P}^{2}\left({ }^{1} \mathrm{D}\right) \\
1 \mathrm{~s}^{2} 2 \mathrm{~S}^{2} 2 \mathrm{P}^{2}\left({ }^{1} \mathrm{~S}^{\circ}\right)\end{array}$ & \\
\hline & & $24^{*}$ & $\begin{array}{l}1 \mathrm{~s}^{2} 2 \mathrm{~S}^{2} \mathrm{P}^{3}\left({ }^{5} \mathrm{~S}^{\bullet}\right) \\
1 \mathrm{~s}^{2} 2 \mathrm{~S} 2 \mathrm{P}^{3}\left({ }^{3} \mathrm{D}^{\bullet}\right) \\
1 \mathrm{~s}^{2} 2 \mathrm{~S} 2 \mathrm{P}^{3}\left({ }^{3} \mathrm{P}^{\circ}\right)\end{array}$ & 6 \\
\hline $\mathrm{N}(\mathrm{III})$ & 6 & 12 & $1 \mathrm{~s}^{2} 2 \mathrm{~S} 2 \mathrm{P}^{2}\left({ }^{4} \mathrm{P}_{\bullet}\right)$ & 1 \\
\hline $\mathrm{N}(\mathrm{IV})$ & 1 & 12 & $\begin{array}{l}1 \mathrm{~s}^{2} 2 \mathrm{~S} 2 \mathrm{P}\left({ }^{3} \mathrm{P}^{\circ}\right) \\
1 \mathrm{~s}^{2} 2 \mathrm{~S} 2 \mathrm{P}\left({ }^{1} \mathrm{P}^{\circ}\right)\end{array}$ & 2 \\
\hline
\end{tabular}

* Four lumped levels used to increase accuracy in the calculation of NII.

From the table presented above, the atomic PF can be calculated. The internal partition function of nitrogen's and oxygen's atom and their derivative are shown separately in Fig. 2(a) and (b). In addition, our outcome using Giuliano's theory made a comparison with existing literature and the simplified method, which neglects the hydrogen-like levels. Both Fig. 2(a) and (b) confirm that our outcome has a good coincidence with the reference [18] which employs an improved cut-off criterion scheme. Relative to the cut-off criterion scheme, this approach significantly accelerates the procedure running time and ensures the accuracy. Thus, with respect to the problem that this paper addresses, the PF's value calculated by the Giuliano method could take the place of the traditional calculations obtained by direct summation over a complete set obtained through Ritz-Rydberg extrapolations [18].

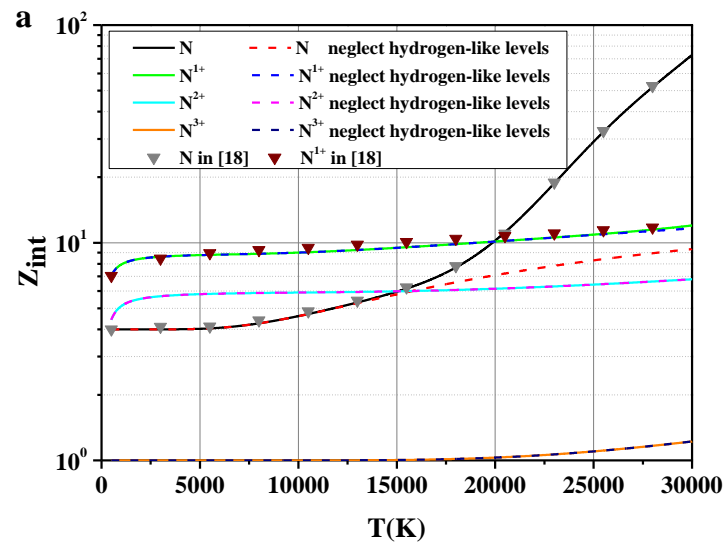

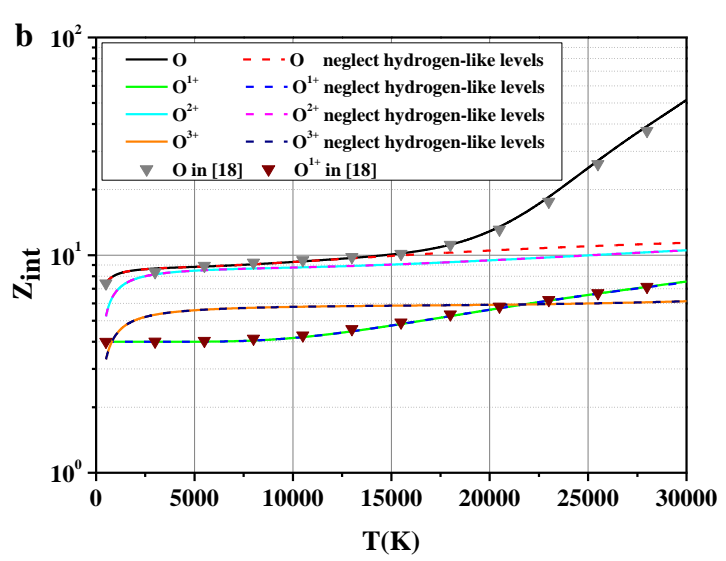

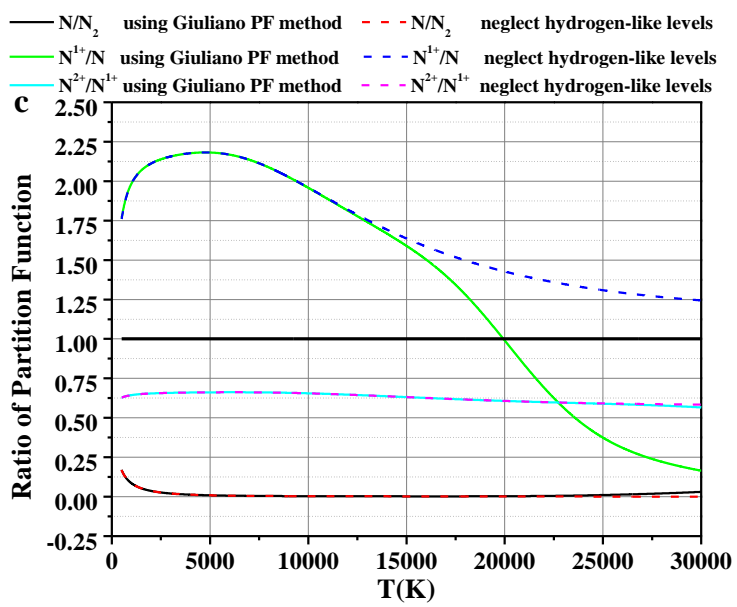

Fig. 2. (a) The partition function of the nitrogen atom and derivative compared with neglected hydrogen-like levels and Ref [18] at 1 atm. (b) The partition function of the oxygen atom and derivative compared with neglected hydrogen-like levels and Ref [18] at $1 \mathrm{~atm}$. (c) Partial PF ratios in mass action law compared to assuming PF's ratio as 1 at $1 \mathrm{~atm}$.

In Fig. 2(a) and (b), the difference for the atoms between the Giuliano PF disposal method and neglecting hydrogen-like levels becomes gradually significant as the temperature rises. As an example for nitrogen atoms, the difference is only $1 \%$ at the temperature of $13450 \mathrm{~K}$, which increases to $10 \%$ at 17230 $\mathrm{K}$, and finally reaches to nearly $90 \%$ at $30000 \mathrm{~K}$. This is because the neglected unknown states are excited as the temperature rises. For ions, the difference between the two methods almost doesn't emerge except for monovalent cations. The difference in $\mathrm{N}^{1+}$ starts to exceed $1 \%$ for almost $27140 \mathrm{~K}$. As a consequence of the differences mentioned above, particles with significant error in PF would cause their calculated concentration to be unreliable at specific 
temperature intervals.

The partial PF ratios of adopting the Giuliano PF method and treating PF's ratio as 1 are shown in Fig. 2(c). As seen in Fig. 2(c), only during the interval from $17500 \mathrm{~K}$ to $22500 \mathrm{~K}$ is the main reactive particles' ratio, which is $Z_{N 1+} / Z_{N}$ calculated by the Giuliano PF method, close to 1 . Consequently, the ratio deviating from the outcome of the Giuliano PF method in other temperature intervals would bring in an inaccurate factor to the composition of electron, ion, and neutral particles. Next the above three diagrams will be applied to analyze the differences of the particle density and refractive index.

\subsubsection{Molecular Internal Partition Function}

The molecular partition function is much more complex than the atom partition function. To simplify the calculation procedure, Mayer's [19] model is employed to calculate symmetrical diatomic gas, which is given by:

$$
\begin{aligned}
\mathrm{Z}_{\text {int }}= & \frac{1}{2} \sum_{\mathrm{e}} \mathrm{g}_{\mathrm{e}} \mathrm{e}^{-\frac{\mathrm{E}_{\mathrm{e}}}{\mathrm{kT}}}\left\{\frac { 1 } { \sigma ( 1 - \mathrm { e } ^ { - \mathrm { u } } ) } \left[1+\frac{\sigma}{3}+\frac{\sigma^{2}}{15}+\frac{8 \gamma^{2}}{\sigma}-\frac{\delta_{\mathrm{e}}}{\left(1-\mathrm{e}^{\mathrm{u}}\right)}+\right.\right. \\
& \left.\left.\frac{2 \chi_{\mathrm{e}} \mathrm{u}}{\left(1-\mathrm{e}^{\mathrm{u}}\right)^{2}}\right]\right\}
\end{aligned}
$$

where

$$
\begin{aligned}
& \delta_{\mathrm{e}}=\frac{\alpha_{\mathrm{e}}}{\mathrm{B}_{\mathrm{e}}} \\
& \sigma=\frac{\left(1-\frac{1}{2} \delta_{\mathrm{e}}\right) \mathrm{B}_{\mathrm{e}} \mathrm{hc}}{\mathrm{kT}} \\
& \mathrm{u}=\frac{\left(1-2 \chi_{\mathrm{e}}\right) \omega_{\mathrm{e}} \mathrm{hc}}{\mathrm{kT}} \\
& \gamma=\frac{\mathrm{B}_{\mathrm{e}}}{\omega_{\mathrm{e}}}
\end{aligned}
$$

All the factors $\delta_{e}, \sigma, u$, and $\gamma$ are different for each electronic state, so they must be calculated at each electronic state, and a summation over all electronic levels must be made. Nitrogen and oxygen, with their derivative's partition functions, are shown in Fig. 3.

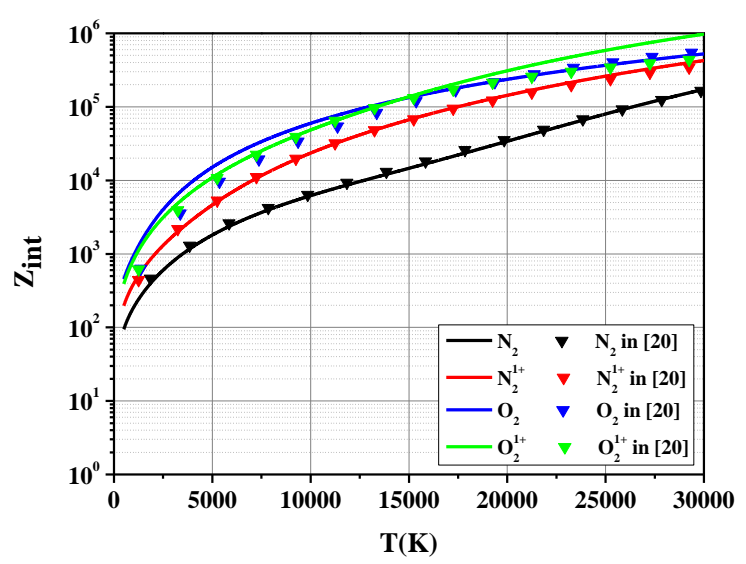

Fig. 3 The partition function of nitrogen and oxygen with their derivatives

As seen in Fig. 3, this paper's result coincides with Drellishak's outcome for nitrogen [20] but a little different for oxygen. That's because Drellishak adopted another method in oxygen's calculation, which has a different disposal means at low excited levels.

\subsection{Plasma's composition and refractive index}

In this paper, the temperature interval is set from $500 \mathrm{~K}$ to $30000 \mathrm{~K}$. The selection of this interval is chosen because most parts of glow discharge and arc discharge are located in this temperature region. Meanwhile, these two kinds of discharges are the research hotspots of air plasma [21]. The pressure of air plasma is set as $1 \mathrm{~atm}$ to calculate the different particle density and the variation of the refractive index versus temperature. The particle density of nitrogen and oxygen calculated by adopting Giuliano's PF disposal method are shown in Fig. 4(a) and (b). 

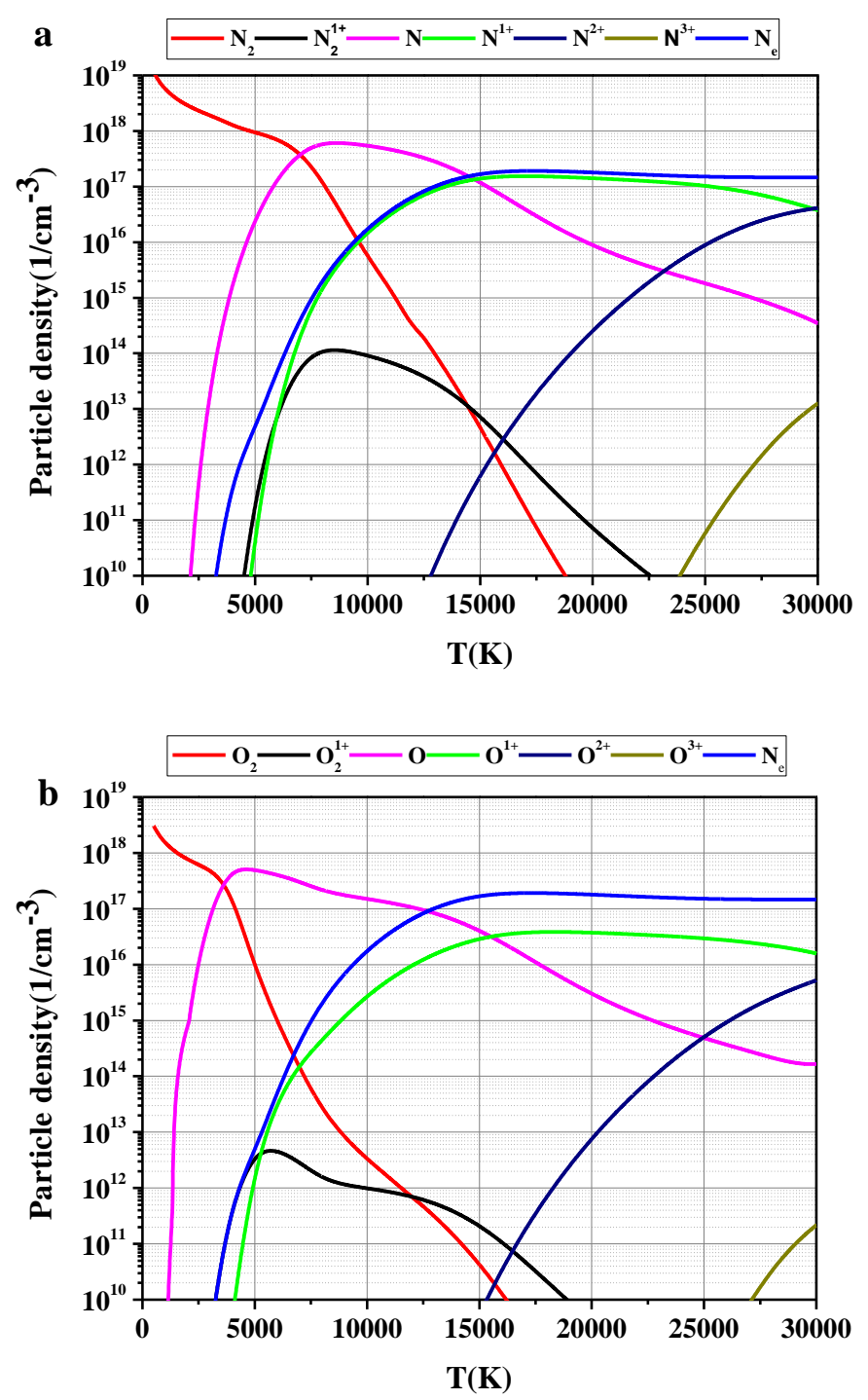

Fig. 4. (a) The particle density of nitrogen solved by Eqs. (1)-(7). (b) The particle density of oxygen solved by Eqs. (1)-(7).

The data of Fig. 4(a) and (b) demonstrate that below 7500 $\mathrm{K}$ dissociation is a leadership reaction to all of the chemical reactions. Because of the assumption of the LTE state for plasma, the dissociation is overestimated in the relatively low temperature region. As the temperature rises up, ionization gradually becomes the dominant reaction. As the diagram shows, nitrogen dissociates from the nitrogen atom, Nitrogen ionizes to the nitrogen ion, and the high-order's ionization happens in sequence. the same goes for oxygen. The sequences and degrees of ionization and dissociation depend on the particles' dissociation or ionization energy.

The number densities of electron, ion, and neutral particles, obtained by adopting different PF disposal methods, are shown in Fig. 5. As shown in Fig. 5(a) and (b), the $\mathrm{N}_{\mathrm{e}}$ and $\mathrm{N}_{\mathrm{i}}$ values gradually decrease from $17320 \mathrm{~K}$ because the thermal expansion of the gas overrides ionization. The decrease of $\mathrm{N}_{\mathrm{e}}$ slows down at about $22500 \mathrm{~K}$, where electrons generated by second and third ionization appear gradually. The differences in the value of $\mathrm{N}_{\mathrm{e}}$, as obtained by treating PF's ratio as 1 , and that calculated from the Giuliano PF methodm is below $1 \%$ in the temperature interval from $16940 \mathrm{~K}$ to $22180 \mathrm{~K}$. Comparing the results of $\mathrm{N}_{\mathrm{e}}$ from the Giuliano PF method with the values obtained by neglecting hydrogen-like levels, the difference between them raises above $1 \%$, only near the peak from $15240 \mathrm{~K}$ to $20440 \mathrm{~K}$. The same difference also appears in the case of $\mathrm{N}_{\mathrm{i}}$.

In Fig. 5(c) it can be seen that the neutral particle's density always decreases because of the increase in the ionization with the rise of temperature. The data obtained by treating PF's ratio as 1 , and by the Giuliano PF method, deviate beyond $1 \%$ from $11590 \mathrm{~K}$. Later the deviation appears in the data by neglecting hydrogen-like levels at $14370 \mathrm{~K}$, too. Finally, at $30000 \mathrm{~K}$, the errors increase up to $86 \%$ for both simplified methods.

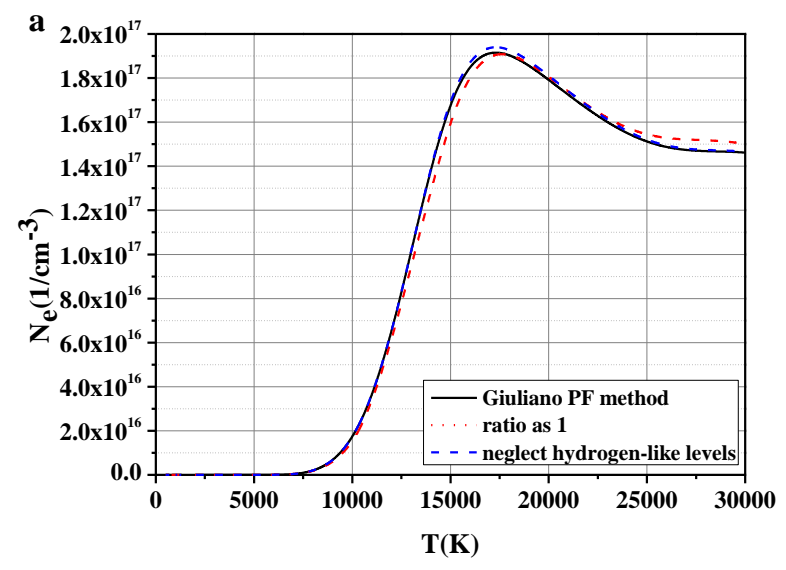



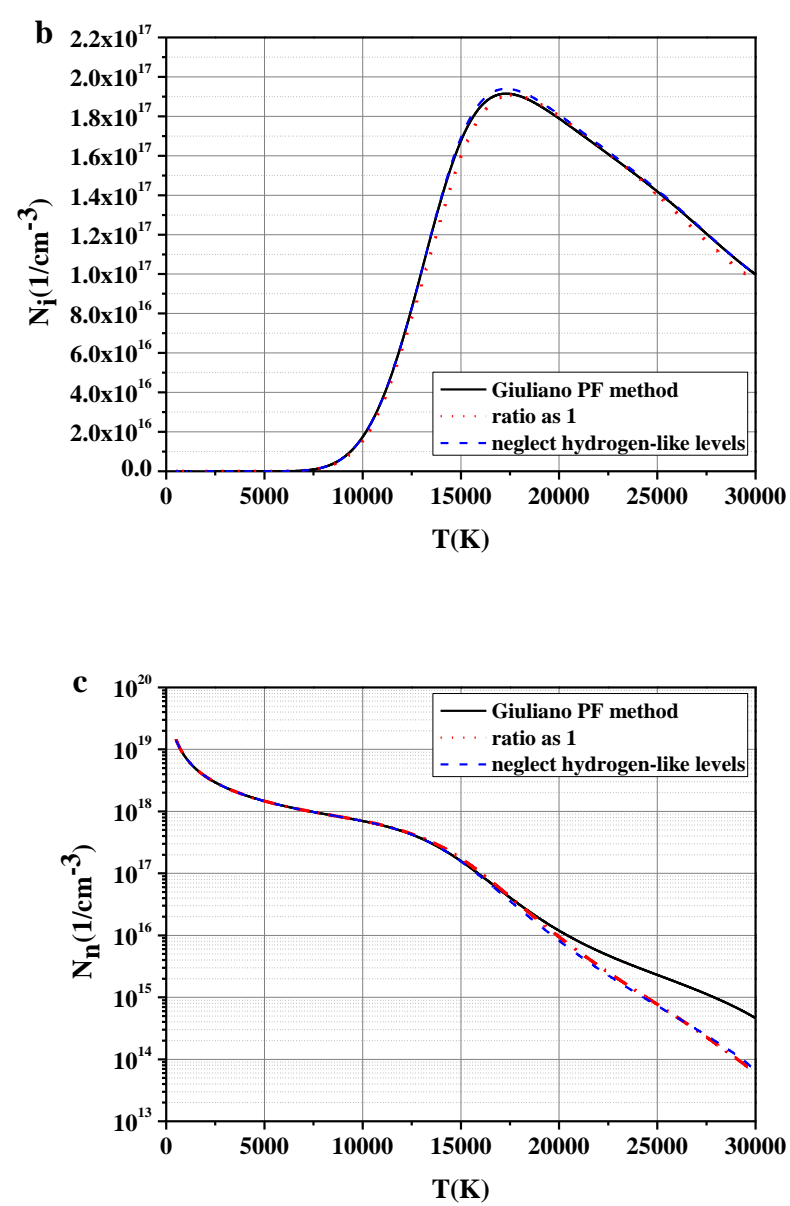

Fig. 5. (a) The relation between the electron density and temperature at 1 atm calculated by three different PF disposal methods. (b) The relation between the ion density and temperature at 1 atm calculated by three different PF disposal methods. (c) The relation between the neutral particle and temperature at 1 atm calculated by three different PF disposal methods.

When comparing the above-mentioned deviation with those discussed along with Fig. 2, both the present errors in $\mathrm{N}_{\mathrm{e}}$ and $\mathrm{N}_{\mathrm{i}}$, when adopting the two simplified PF methods, coincide with the same temperature intervals of the error in Fig. 2. However, the error of $\left(\mathrm{Z}_{\mathrm{N}}\right)^{2} / \mathrm{Z}_{\mathrm{N} 2}$ in Fig. 2(c) doesn't emerge on $\mathrm{N}_{\mathrm{n}}$ in the low temperature region of Fig. 5(c). A reasonable explanation for $\mathrm{N}_{\mathrm{n}}$ 's error not appearing when treating PF's ratio as 1 is that the combination of the dominant particles' PF ratio and the value of exponential terms play a key role in the mass action law. When the exponential term's order of magnitude is much smaller than the ratio's order of magnitude, the error of treating PF's ratio as 1 does not emerge.
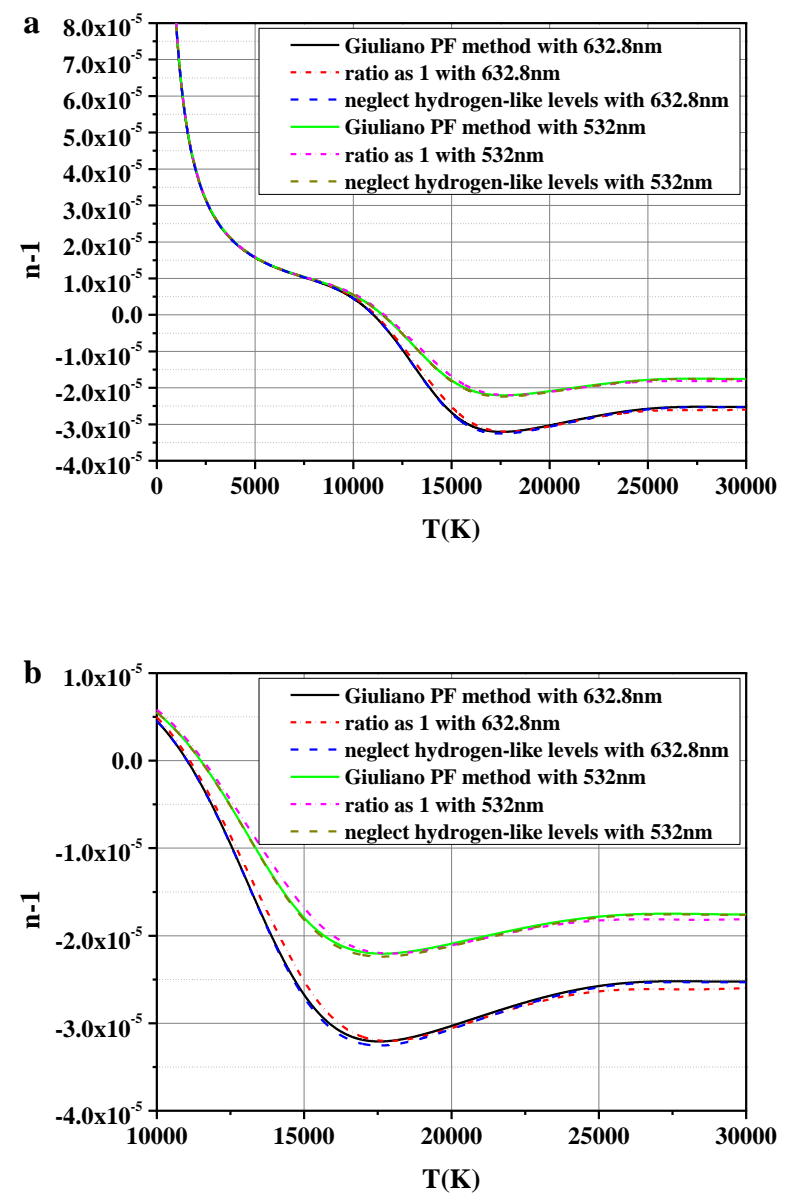

Fig. 6. (a) Distribution of $n-1$ versus temperature at probe wavelength $632.8 \mathrm{~nm}$ and $532 \mathrm{~nm}$ with pressure $1 \mathrm{~atm}$ by using different PF disposal methods. (b) The enlargement of the deviation between three methods from $10000 \mathrm{~K}$ to $30000 \mathrm{~K}$.

Fig. 6 presents the difference in the variation of the refractive index (n-1) versus temperature at probe wavelength $632.8 \mathrm{~nm}$ obtained by adopting the three PF disposal methods. Another probe wavelength at $532 \mathrm{~nm}$ evaluated by employing the three different PF methods are also plotted. It can be seen that in the low temperature region there is no difference caused by different probe wavelengths and PF disposal methods. The reason for this is that the neutral particle's density has a substantial weight at low temperatures, as shown in Eq. (8) and Fig. 5. In addition, by adopting the Giuliano PF method, the deviation between $532 \mathrm{~nm}$ and $632 \mathrm{~nm}$ starts to emerge at about $7180 \mathrm{~K}$. With the increase of temperature and the decrease of the neutral particle's density, the wavelength's coefficient becomes more considerable compared to the neutral particle's density. 
As the temperature rises, the weight of electrons in n-1 gradually increases. The method by treating PF's ratio as 1 exhibits an error below $1 \%$ only when the temperature is below $8600 \mathrm{~K}$, and in the interval from $17040 \mathrm{~K}$ to $21410 \mathrm{~K}$. For the other method, the neglect of hydrogen-like levels, the error is always below $1 \%$ except for the intervals from 10910 $\mathrm{K}$ to $11410 \mathrm{~K}$ and from $14680 \mathrm{~K}$ to $21420 \mathrm{~K}$. The former is the zero crossing point of $n-1$, while the latter is the particles dominated by $\mathrm{N}$ and $\mathrm{N}^{1+}$. Similar error variation appears at the wavelength of $532 \mathrm{~nm}$ too. The only difference is that the zero crossing point of $532 \mathrm{~nm}$ occurs a little later.

Considering Eq. (8), the errors of $n-1$ by adopting different PF methods are caused by the errors of $\mathrm{N}_{\mathrm{e}}, \mathrm{N}_{\mathrm{i}}$, and $\mathrm{N}_{\mathrm{n}}$, as $\mathrm{n}-1$ is a function of the three types' of particle number densities as mentioned above. These results indicate that, although treating the ratio as 1 is the most simplified method towards PF, it is only satisfactory in narrower temperature intervals than the approach of neglecting hydrogen-like levels. If the simplification is used impertinently in $n-1$, the error of n-1 caused by PF may affect the accuracy of the temperature diagnosis finally.

\section{Experiment and results}

For a better understanding of the PF's influences on the temperature diagnosis, experimental set-up of moiré deflection is constructed. The schematic of the experimental set-up is shown in Fig. 7. A solid-state laser with wavelength of $532 \mathrm{~nm}$ and maximum power of $600 \mathrm{~mW}$ is used as a probe light source. Plano-convex lens L1 and L2 are used to expand and collimate the light beam. Two Ronchi gratings with the pitch $d=0.05 \mathrm{~mm}$ are used. The distance between two gratings is equal to $164 \mathrm{~mm}$. Grating $G_{1}$ and $G_{2}$ have a small angle to each other to generate moiré fringes. Plano convex lens $\mathrm{L}_{3}$ and $\mathrm{L}_{4}$ construct the imaging system to map the fringes formed at $G_{2}$ onto the camera. In this experiment, the surrounding atmosphere's refractive index is estimated as 1.00027, and the air pressure is assumed to be $1 \mathrm{~atm}$. The discharge-produced plasma is generated under the condition that the transformer's voltage is boosted to $10 \mathrm{kV}$, across a pair of needle copper electrodes. The effective value of the AC current is about $100 \mathrm{~mA}$. An image of the discharge plasma by a digital camera is shown in Fig. 8. Exposure time of the picture is set at about $250 \mathrm{~ms}$, and a bright elliptic profile of the plasma can be found.

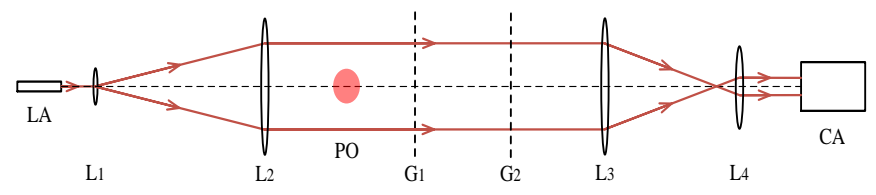

Fig. 7. Schematic diagram of moiré deflectometry

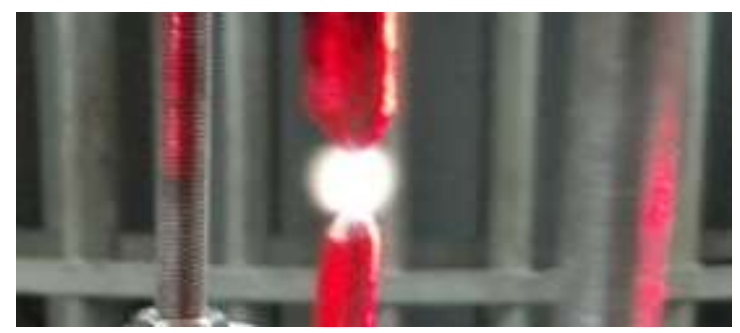

Fig. 8. Image of discharge plasma with an exposure time of $250 \mathrm{~ms}$

Fig. 9 shows the moiré deflection and reference fringe patterns. The distributions of the $n-1$ versus distance from the air plasma center pointed by the five colorized arrows are shown in Fig. 10. From the moiré deflection fringe in Fig. 9, plasma's phase difference can be obtained by disposing deflection fringe with the reference based on a Fast Fourier transform method. The purpose of acquiring the phase difference is to further deduce the deflection angle. Then by using the Abel inversion on the deflection angle, the distribution of the refractive index versus distance pointed by the arrow could finally be obtained [22].

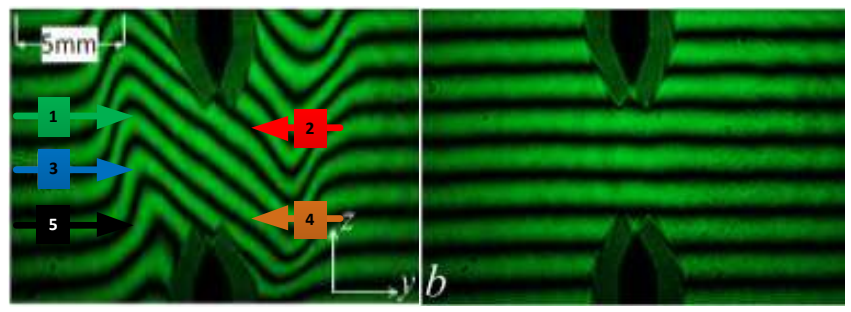

Fig. 9. Deflection fringe patterns and reference patterns

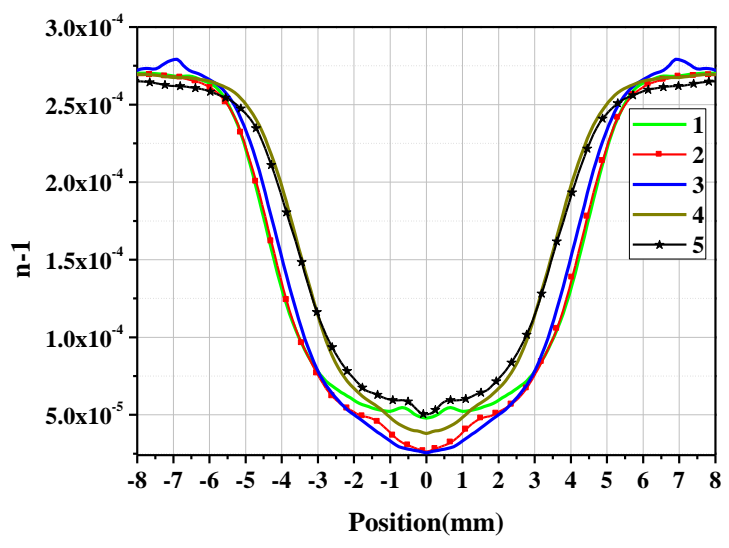


Fig. 10. The distribution of the $n-1$ versus distance from the air plasma center indicated by the colorized arrows in Fig. 9

Fig. 11 shows the temperature distribution versus distance from the air plasma center deduced from the refractive index in Figs. 6 and 10. It should be mentioned that the distribution of the $n-1$ versus temperature is extended to $300 \mathrm{~K}$ to deduce the surrounding air's temperature. As shown in Fig. 11(a), the highest temperature is at the plasma's main axis, and a rapid reduction would occur from the center to both sides. Examining the outcome of two simplified PF methods in Fig. 11(b), both of them coincide with that of the Giuliano PF method because both of them show low error in the low temperature region, as shown in Fig. 6.
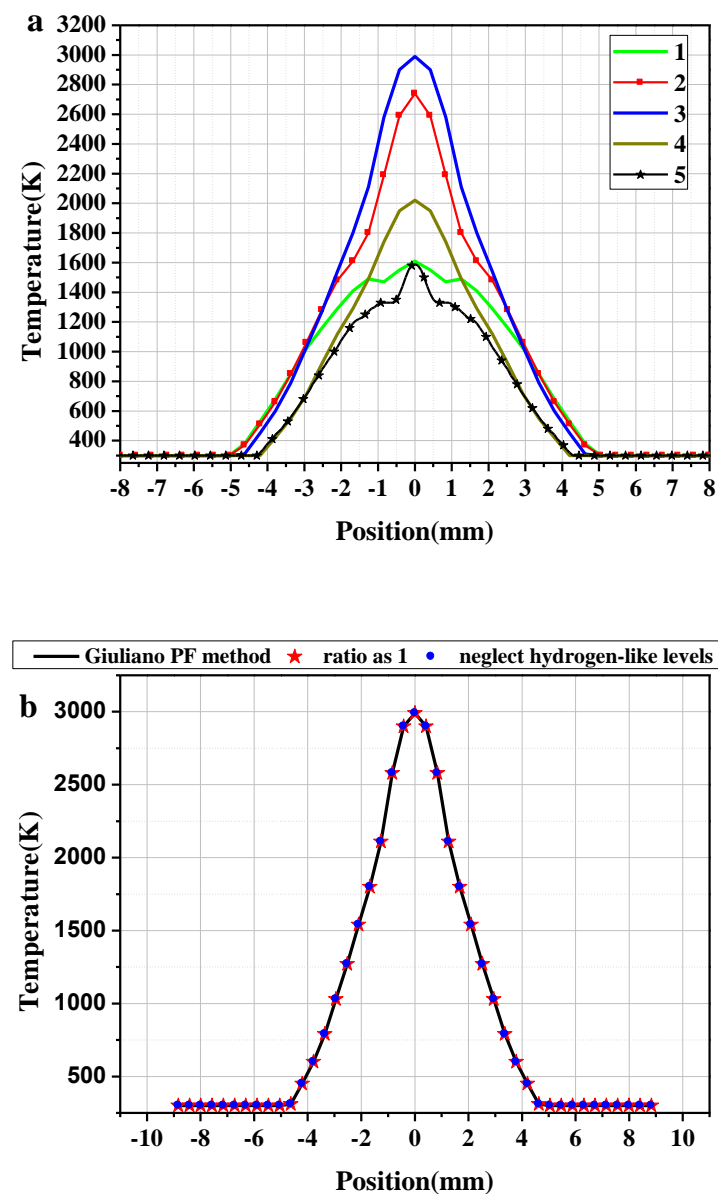

Fig. 11. (a) The temperature distribution deduced from Fig. 6 (a) by adopting the Giuliano PF method. (b) The temperature distribution at layer 3 deduced from Fig. 6(a) by using different PF disposal methods.

Fig. 12 is the emission spectrum of the plasma at layer 3. The detailed schematic diagram of spectral experiments is explained in previous work [23] and would not be repeated here. From Fig. 12 it can be seen that our temperature distribution obtained in Fig. 11 coincides well with the data calculated by spectral analysis.

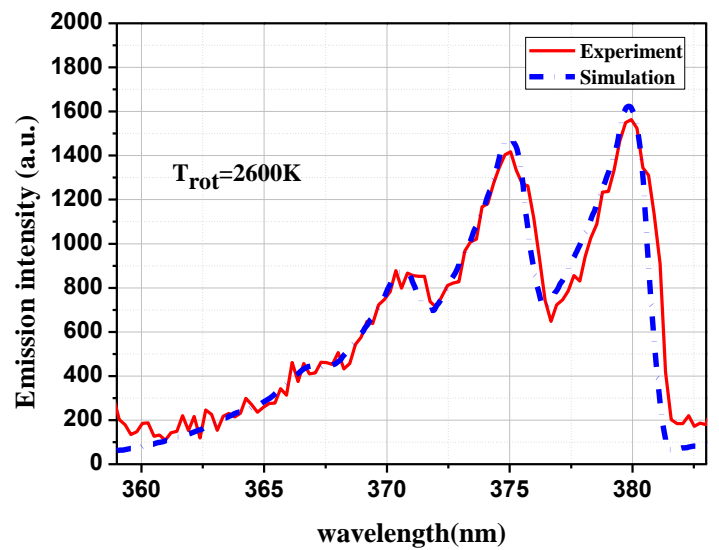

Fig. 12. The emission spectrum of the plasma at layer 3

For a further understanding of the temperature distribution influenced by PF, we would like to analyze the distribution of the refractive index versus position in Ref [6], which employs a similar moiré deflectometry experimental setup to diagnose pulsed discharge-produced plasmas. We used the original data from Ref. [6] and our own disposal scheme to deduce the final temperature profile. As shown in Fig. 13, the difference in temperature distribution between treating PF's ratio as 1 and the Giuliano PF method emerges more evidently. In the main axis the error of temperature distribution is about $0.81 \%$ at $11100 \mathrm{~K}$. The error gradually decreases as the horizontal position deviates from the main axis, which is because the error in the curves of the $n-1$ versus temperature decreases at the relative lower temperature interval. Also our data is compared to the outcome in Ref. [6]. The differences may be caused by the different PF's disposal method and the inversion method for the deflection angle. 


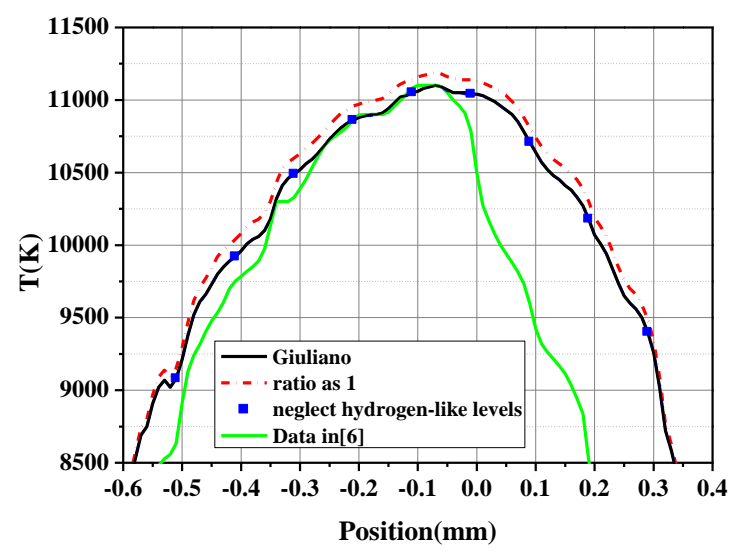

Fig. 13. The temperature distribution deduced from Ref. [6] by using different PF disposal methods.

As shown in the diagrams of temperature diagnosis, the accuracy of temperature distribution is dependent on the error in the curves of the $\mathrm{n}-1$ versus temperature. After making a comparison within the three PF disposal methods, the Giuliano PF method is always well suited in the whole temperature interval for refractive index calculation and temperature diagnosis. Another simplified method by neglecting hydrogen-like levels also coincides with the Giuliano PF method, except for the intervals dominant by primary ionization. The least applicable simplified method is treating PF's ratio as 1. Although it reduces the equations' order and consumes the least running time, it satisfies the fewest temperature intervals in the refractive index calculation and temperature diagnosis.

\section{Conclusion}

In this paper, we used a simplified and compact method to calculate the atomic partition function, eschewing the time-consuming scheme of cut-off criterion. The outcome coincides well with existing references. Besides, we compared the proposed method with the other two traditional schemes, where hydrogen-like levels are neglected and PF's ratio is treated as a constant unit respectively. Results indicate that our proposed method satisfied accuracy in the range of $500 \mathrm{~K}$ to $30000 \mathrm{~K}$ for refractive index temperature curves, while the other two traditional schemes are only validated in limited temperature intervals.

After the establishment of relationships between the optical properties with plasma parameters, we used the moiré deflectometry technique to deduce the temperature of typical discharge-produced plasmas. Image and optical emission spectroscopy of the plasmas have also been presented. Results indicate that the optical properties deduced in this paper are well suited for the refractive index-based plasma diagnosis.

\section{ACKNOWLEDGMENTS}

The authors would like to gratefully acknowledge Professor Anthony B. Murphy from CSIRO Materials Science and Engineering, Australia for his valuable technical suggestions in the calculation of molecular partition function. Project supported by Natural Science Foundation of China (U1234202 ，51577158 ，51607147) ， National Science Foundation for Distinguish Young Scholars of China (51325704).

\section{REFERENCE}

[1] Schutze A, Jeong J Y, Babayan S E, et al. IEEE transactions on plasma science, 26 (6) (1998) 1685-1694.

[2] T. Shimizu, R. Pompl, B. Steffes, F. Jamitzky, W. Bunk, K. Ramrath, M. Georgi, G. Isbary, W. Stolz, H.-U. Schmidt, T. Urayama, S. Fujii, G.E. Morfill Max Planck Institute for Extraterrestrial Physics, Proceeding, No.2 (2008)

[3] Meidanshahi, Fatemeh Salimi, K. Madanipour, and B. Shokri. Optics Communications 285 (4) (2012) 453-458.

[4] Rong M Z, Wu Y, Fei Y, Sun Z Q, Wang W Z, Wang X H, Proceeding of the XVII International Conference on Gas Discharges and Their Applications Cardiff, UK, 2008.

[5] Chen, Yunyun, et al. Applied Optics 48 (3) (2009) 489-96. [6] Meidanshahi, Fatemeh Salimi, K. Madanipour, and B. Shokri, Optics\&Lasers in Engineering 51 (4) (2013) 382-387. [7] C. Yun-yun, L. Zhen-hua, S. Yang, H. An-zhi, Applied Optics 48 (13) (2009) 2485.

[8] Murphy, Anthony B, Chemical Physics 398 (1) (2012) 64-72. 
[9] Richley, E, and D. T. Tuma, Journal of Applied Physics 53

(12) (1983) 8537-8542.

[10] D'Ammando, Giuliano, G. Colonna, and M. Capitelli, Physics of Plasmas 20 (3) (2013) 184-187.

[11] Colonna, G., and M. Capitelli, Spectrochimica Acta Part B Atomic Spectroscopy 64 (9) (2009) 863-873.

[12] D'Ammando, G., et al, Spectrochimica Acta Part B Atomic Spectroscopy 65 (8) (2010) 603-615.

[13] Boulos, Maher I., P. Fauchais, and E. Pfender. Thermal Plasmas: Fundamentals and Applications, Vol. 1. Thermal plasmas: fundamentals and applications, (1994) 1258 - 1280.

[14] Sanden, M. C. M. Van De, et al, Physical Review A 40 (40) (1989) 5273-5276.

[15] Gleizes, A., B. Chervy, and J. J. Gonzalez, Journal of Physics D Applied Physics 32 (16) (1999) 2060-2067.

[16] Griem, H. R. Principles of Plasma Spectroscopy. Fast Electrical and Optical Measurements. Springer Netherlands, (2005) 885-910.

[17] NIST Atomic Spectra Database (version 5.3), http://physics.nist.gov/PhysRefData/ASD/levels_form.html

National Institute of Standards and Technology, U.S. Department of Commerce.

[18] Bacri, J, and S. Raffanel, Plasma Chemistry \& Plasma Processing 7 (1) (1987) 53-87.

[19] Vleck, J. H. Van, J.phys.chem 3 (1941) 539-540.

[20] Drellishak, K. S., D. P. Aeschliman, and A. B. Cambel, Physics of Fluids 8 (9) (1965) 1590-1600.

[21] Keidar, Michael, and I. Beilis. Plasma Engineering. 2013. [22] Kolhe, Pankaj S., and A. K. Agrawal, Applied Optics 48 (20) (2009) 3894-902.

[23] Gao G, Hao J, Wei W, et al, IEEE Transactions on Plasma Science, 44 (11) 2016, 2715-2723. 